\title{
A test for the equality of multiple Sharpe ratios
}

\section{John Alexander Wright}

Department of Statistics, The Chinese University of Hong Kong, Shatin, Hong Kong; email: jawright@sta.cuhk.edu.hk

\section{Sheung Chi Phillip Yam}

Department of Statistics, The Chinese University of Hong Kong, Shatin, Hong Kong; email: scpyam@sta.cuhk.edu.hk

\section{Siu Pang Yung}

Department of Mathematics, The University of Hong Kong, Hong Kong; email: spyung@hkucc.hku.hk

(Received April 18, 2011; revised September 7, 2012; accepted September 7, 2012)

\begin{abstract}
This paper provides a test for the equality of multiple Sharpe ratios. First we extend the multivariate Sharpe ratio statistic of Leung and Wong for the case when excess returns are independently and identically distributed. We then provide a test that holds under the much more general assumption that the excess returns are stationary and ergodic, making use of the generalized method of moments and heteroscedasticity and autocorrelation consistent estimation of covariance matrixes. We repeat Leung and Wong's testing for equality of the Sharpe ratios of 18 iShares using our new tests and conclude that the hypothesis of equality cannot be rejected at the $1 \%$ level.
\end{abstract}

\section{INTRODUCTION}

Sharpe (1994) defined the Sharpe ratio (SR) as:

$$
\mathrm{SR}=\frac{\mathbb{E}\left[R_{F}-R_{B}\right]}{\left(\operatorname{Var}\left[R_{F}-R_{B}\right]\right)^{1 / 2}}
$$

We are grateful to the anonymous referees and many seminar and conference participants for their valuable comments and suggestions. John Wright expresses his thanks to the University of Hong Kong and the Chinese University of Hong Kong for hosting him as a doctoral student and a visiting academic, respectively, during which time much of his work on this paper was done. Phillip Yam acknowledges financial support from the HKSAR-RGC-GRF (grant 404012) under a project title Advanced Topics in Multivariate Risk Management in Finance and Insurance, and from direct grants 2060422 and 2060444; he would also like to express sincere gratitude to the hospitality of the Chinese Academy of Science, the Hausdorff Center for Mathematics of the University of Bonn, and Mathematisches Forschungsinstitut Oberwolfach during the preparation of the manuscript. S. P. Yung acknowledges support from an internal University of Hong Kong grant (201109176016). 
where $R_{F}$ is the arithmetic return on fund/stock/asset $F$ over one time interval and $R_{B}$ is the arithmetic return on benchmark asset $B$ over the same interval. Subtracting $R_{B}$ from $R_{F}$ gives the differential (or excess) return, $d$. SR, then, is the expected value of $d$ divided by its standard deviation. This definition is an improvement on the first version of the SR, introduced in Sharpe (1966) and then called the rewardto-variability ratio. The more recent definition takes into account the possibility that $R_{B}$ may vary over time.

Sharpe (1994) gives several examples where choosing the best fund to invest in (in terms of maximizing the return on investor's assets) is equivalent to choosing the fund with the highest SR. One example is when the investor has cash in a bank account and the benchmark asset is a risk-free bond. However, other examples where the SR is not sufficient to decide which fund is best are also given. In particular, if an investor's assets are correlated with each of a selection of funds, choosing the fund with the highest SR may not be optimal (Sharpe 1994). Nevertheless, Sharpe ratios are commonly used as arguments for (or against) investing in a fund or asset.

Hypothesis tests for the equality of funds' Sharpe ratios depend critically on the assumptions made of the excess returns. Jobson and Korkie (1981) developed pairwise and multivariate tests for equality assuming that the funds' excess returns are well modeled by a multivariate normal distribution. Memmel (2003) later improved their pairwise test, again assuming normality. Ledoit and Wolf (2008) provide a pairwise test that, via a bootstrap approach, allows for returns of a time series nature and returns with heavy tails. As such, their test is of greater practical use than the previous pairwise tests. Leung and Wong (2008) sought a multivariate test that only assumes excess returns to be independent and identically distributed (iid), but we shall see that their test statistic is valid only under the assumption of normality. In this paper we will address this issue and present the correct test statistic.

In addition, we will also provide a test that requires the excess returns to be stationary and ergodic and which therefore permits the returns to exhibit properties that, although violations of the iid assumption, are common to financial returns, such as autocorrelation and conditional heteroscedasticity. We achieve this by employing the generalized method of moments estimators of Hansen (1982), an approach to creating hypothesis tests for Sharpe ratios that was outlined in Lo (2002).

The funds we use to illustrate our test for equality of multiple Sharpe ratios are those used in Leung and Wong (2008) and Gasbarro et al (2007), namely 17 iShares and Standard \& Poor's depository receipts. The iShares began trading on the American Stock Exchange in March 1996 as world equity benchmark shares and are designed to track the Morgan Stanley Capital International foreign stock market indexes. The Standard \& Poor's depository receipts track the S\&P 500 index and began trading in January 1993 and shall be used as an American stock market index.

Journal of Risk 16(4) 
Gasbarro et al (2007) administered the pairwise test for equality of Sharpe ratios of Memmel (2003) on all possible pairs on the 18 tradable securities and found that "none of the Sharpe Ratios are significantly different". Leung and Wong (2008) repeated these tests and drew the same conclusion. However, they also applied their multivariate test for equality to the same data set and concluded that "the Sharpe ratios of some iShares are different from the others for each year as well as the entire sample". We shall test for the equality of the Sharpe ratios a third time, using the updated and more general version of Leung and Wong's (2008) multivariate test.

This paper is structured as follows. In Section 2 we derive the asymptotic distribution of multiple Sharpe ratios, under the assumption that the excess returns of each fund are iid. The relevant calculations are given in Appendix A, which also shows how our results reduce to existing results when we consider only one stock and to the test of Leung and Wong (2008) when returns are multivariate normal. In Section 3, we derive the hypothesis test for the equality of multiple Sharpe ratios in the iid case, following Leung and Wong's (2008) lead, itself following a standard statistical procedure found in Johnson and Wichern (2007). Also included is a simulation study that demonstrates the inappropriateness of Leung and Wong's (2008) test when returns are nonnormal. In Section 4, we use the generalized method of moments of Hansen (1982) and the heteroscedasticity and autocorrelation robust (HAC) inference methods outlined in Section 3.1 of Ledoit and Wolf (2008) in the bivariate case to develop a hypothesis test that does not require excess returns to be iid. We apply this test to the 17 iShares and depository receipts used in Leung and Wong (2008) and Gasbarro et al (2007). Finally, in Section 5 we summarize the results and conclude.

\section{ASYMPTOTIC DISTRIBUTION OF THE SHARPE RATIO}

Before the distribution of the Sharpe ratio can be derived, assumptions must be made about the distribution of the underlying random variable of which the Sharpe ratio is a function: the excess return.

We shall consider $k$ funds and their daily excess returns over the past $n$ days. Fund $i$ has excess return $X_{i t}$ on day $t$. Consider the sample mean of the excess returns and the sample mean of the squared excess returns for fund $i$ :

$$
\hat{m}_{1}^{i}:=\frac{1}{n} \sum_{j=1}^{n} X_{i j} \quad \text { and } \quad \hat{m}_{2}^{i}:=\frac{1}{n} \sum_{j=1}^{n}\left(X_{i j}\right)^{2},
$$

which are unbiased estimators for the first and second moments $\left(m_{1}^{i}\right.$ and $\left.m_{2}^{i}\right)$ of $X_{i t}$ respectively. We shall assume that the daily $k$-variate vectors of excess returns are iid and that the daily excess return for each fund has a finite fourth moment. These conditions are general enough to include a wealth of return models (including Lévy processes) and are required in order for the central limit theorem to be used. 
The multivariate central limit theorem states that if $\underline{Y}_{1}, \underline{Y}_{2}, \ldots, \underline{Y}_{n}$ are vectors of independent observations from any population with mean vector $\mu$ and finite covariance matrix $\underline{\Sigma}$ then

$$
\sqrt{n}(\bar{Y}-\underline{\mu}) \stackrel{\mathrm{D}}{\rightarrow} \mathrm{N}(\underline{0}, \underline{\Sigma}),
$$

where $\bar{Y}$ is the sample mean vector and $\stackrel{\mathrm{D}}{\rightarrow}$ represents asymptotic convergence in distribution.

With the above result in mind, we define the $2 k \times 1$ vector $\underline{X}_{t}$ which has entries $X_{i t}$ for rows 1 to $k$ and $X_{i t}^{2}$ for rows $k+1$ to $2 k$. Thus $\underline{X}_{t}$ contains the historical excess returns and squared excess returns for all funds on day $t$. Our assumptions for the behavior of the excess returns permit the use of the multivariate central limit theorem, which, in turn, tells us the asymptotic distribution of the sample mean vector $\left(\bar{X}_{n}\right)$ of $\underline{X}_{t}$. In this case, mean vector $\mu$ has entries $m_{1}^{i}$ for rows 1 to $k$ and $m_{2}^{i}$ for rows $k+1$ to $2 k$. Covariance matrix $\underline{\Sigma}$ may be written in terms of three $k \times k$ submatrixes $A, B$ and $C$ where

$$
\underline{\Sigma}=\left(\begin{array}{cc}
A & C \\
C^{\mathrm{T}} & B
\end{array}\right)
$$

and $A$ is the covariance matrix for the $k$ excess returns, $B$ is the covariance matrix for the $k$ squared excess returns and $C$ is the covariance matrix for the $k$ excess returns and their squares.

The Sharpe ratio for fund $i, \mathrm{SR}_{i}$, is equal to the mean excess return $m_{1}^{i}$ divided by the standard deviation of the excess return $\sqrt{m_{2}^{i}-\left(m_{1}^{i}\right)^{2}}$. As we know the asymptotic distribution of $\bar{X}_{n}$ we may use the multivariate delta method to find the asymptotic distribution of the $k \times 1$ vector of Sharpe ratio estimates. Let $\widehat{\mathrm{SR}}$ be a $k \times 1$ vector with entries $\hat{m}_{1}^{i} / \sqrt{\hat{m}_{2}^{i}-\left(\hat{m}_{1}^{i}\right)^{2}}$ for each row $i$. Thus the $i$ th component of $\widehat{\widehat{S R}}$ is the natural estimator for the Sharpe ratio of fund $i$.

Define vector function $\underline{f}$ by the following:

$$
\begin{aligned}
& \underline{f}: \mathbb{R}^{2 k} \mapsto \mathbb{R}^{k} \text { and for } \underline{x}=\left(x_{1}, x_{2}, \ldots, x_{2 k}\right)^{\mathrm{T}}, \\
& f_{i}:=(\underline{f}(\underline{x}))_{i}:=\frac{x_{i}}{\sqrt{x_{k+i}-x_{i}^{2}}} \text { for } i=1, \ldots, k .
\end{aligned}
$$

It is clear that $\underline{f}\left(\bar{X}_{n}\right)$ is equal to $\underline{\widehat{\mathrm{SR}}}$, since for $i=1, \ldots, k$

$$
f_{i}=\frac{\hat{m}_{1}^{i}}{\sqrt{\hat{m}_{2}^{i}-\left(\hat{m}_{1}^{i}\right)^{2}}}=(\widehat{\mathrm{SR}})_{i},
$$

which is the estimator for the Sharpe ratio of fund $i$. The multivariate delta method states that if $\underline{f}$ has a differential at $\underline{\mu}$ and

$$
\left(\frac{\partial \underline{f}}{\partial \underline{\mu}}\right)_{i j}:=\left.\frac{\partial f_{i}}{\partial x_{j}}\right|_{\underline{x}=\underline{\mu}} \quad \text { for } i=1, \ldots, k \text { and } j=1, \ldots, 2 k,
$$


then the asymptotic distribution of $\underline{f}\left(\bar{X}_{n}\right)$ is given by

$$
\sqrt{n}\left(\underline{f}\left(\bar{X}_{n}\right)-\underline{f}(\underline{\mu})\right) \stackrel{\mathrm{D}}{\rightarrow} \mathrm{N}\left(\underline{0},\left(\frac{\partial \underline{f}}{\partial \underline{\mu}}\right) \underline{\Sigma}\left(\frac{\partial \underline{f}}{\partial \underline{\mu}}\right)^{\mathrm{T}}\right) .
$$

Calculating the entries of the Jacobian matrix, we find that

$$
\left(\frac{\partial \underline{f}}{\partial \underline{x}}\right)_{i j}= \begin{cases}0 & \text { if } j \neq i \text { or } j \neq i+k, \\ \frac{x_{k+j}}{\left(x_{k+j}-x_{j}^{2}\right)^{3 / 2}} & \text { if } j=i, \\ \frac{-x_{j}}{2\left(x_{k+j}-x_{j}^{2}\right)^{3 / 2}} & \text { if } j=i+k,\end{cases}
$$

so it is certainly true that $\underline{f}$ has a differential at $\underline{\mu}$ as each fund has a nonzero standard deviation. Hence the delta method may be applied and we find the asymptotic distribution of our vector of estimates of Sharpe ratios for the $k$ funds:

$$
\sqrt{n}(\underline{\widehat{\mathrm{SR}}}-\underline{\mathrm{SR}}) \stackrel{\mathrm{D}}{\rightarrow} \mathrm{N}\left(\underline{0}, D \underline{\Sigma} D^{\mathrm{T}}\right),
$$

where $D$ is a $k \times 2 k$ matrix

$$
D^{\mathrm{T}}=\left(\begin{array}{cccc}
\frac{m_{2}^{1}}{\left(m_{2}^{1}-\left(m_{1}^{1}\right)^{2}\right)^{3 / 2}} & 0 & \cdots & 0 \\
0 & \frac{m_{2}^{2}}{\left(m_{2}^{2}-\left(m_{1}^{2}\right)^{2}\right)^{3 / 2}} & & \vdots \\
\vdots & & \ddots & 0 \\
0 & \cdots & 0 & \frac{m_{2}^{k}}{\left(m_{2}^{k}-\left(m_{1}^{k}\right)^{2}\right)^{3 / 2}} \\
\frac{-m_{1}^{1}}{2\left(m_{2}^{1}-\left(m_{1}^{1}\right)^{2}\right)^{3 / 2}} & 0 & \cdots & 0 \\
0 & \frac{-m_{1}^{2}}{2\left(m_{2}^{2}-\left(m_{1}^{2}\right)^{2}\right)^{3 / 2}} & & \vdots \\
\vdots & & \ddots & 0 \\
0 & \cdots & 0 & \frac{-m_{1}^{k}}{2\left(m_{2}^{k}-\left(m_{1}^{k}\right)^{2}\right)^{3 / 2}}
\end{array}\right)
$$

It is with respect to the asymptotic covariance matrix $D \underline{\Sigma} D$ that our result differs from Leung and Wong (2008). In their paper, the authors derive the asymptotic distribution for the $2 k \times 1$ vector $\hat{\theta}:=\left(\bar{x}_{1}, \bar{x}_{2}, \ldots, \bar{x}_{k}, s_{1}^{2}, s_{2}^{2}, \ldots, s_{k}^{2}\right)^{\mathrm{T}}$, where $\bar{x}_{i}$ and $s_{i}^{2}$ are the sample mean and variance of the excess returns of fund $i$. Quoting from the paper: 
According to the standard asymptotic distribution theory, we have

$$
\sqrt{n}[\hat{\theta}-\theta] \stackrel{\mathrm{D}}{\rightarrow} \mathrm{N}(0, \Sigma)
$$

where

$$
\Sigma=\left(\begin{array}{cc}
\Sigma_{1} & \mathbf{0} \\
\mathbf{0} & \Sigma_{2}
\end{array}\right)
$$

is a $2 k \times 2 k$ matrix such that

$$
\Sigma_{1}=\left(\begin{array}{ccc}
\sigma_{1}^{2} & \ldots & \sigma_{1 k} \\
\vdots & \ddots & \vdots \\
\sigma_{1 k} & \ldots & \sigma_{k}^{2}
\end{array}\right), \quad \Sigma_{2}=\left(\begin{array}{ccc}
2 \sigma_{1}^{4} & \ldots & 2 \sigma_{1 k}^{2} \\
\vdots & \ddots & \vdots \\
2 \sigma_{1 k}^{2} & \ldots & 2 \sigma_{k}^{4}
\end{array}\right)
$$

and $\mathbf{0}$ is a $k \times k$ matrix of zeros.

However, this is only true if the excess returns for each fund are normally distributed. The zero matrixes in $\Sigma$ correspond to independence of the sample mean and sample standard deviation, a property unique to the normal distribution. Having applied the delta method, Leung and Wong (2008) show that their asymptotic variance agrees with those found in Lo (2002) and Memmel (2003), but both these papers assumed excess returns to be normally distributed. Therefore Leung and Wong (2008) does not provide the asymptotic distribution for multiple Sharpe ratio when excess returns are iid, but only when they follow a multivariate normal distribution. In Appendix A we calculate the entries of $D \underline{\Sigma} D^{\mathrm{T}}$ explicitly and show that they are equivalent to the entries of the covariance matrix in Leung and Wong (2008) only when returns follow a multivariate normal distribution.

\section{A HYPOTHESIS TEST FOR THE EQUALITY OF MULTIPLE SHARPE RATIOS: IID CASE}

In this section we follow Leung and Wong (2008) and derive a hypothesis test for the equality of multiple Sharpe ratios that adheres to a repeated-measures design for comparing treatments, explained in Chapter 6 of Johnson and Wichern (2007). It was proven in the previous section that if the daily excess returns of $k$ different funds are iid with finite fourth moments, then when $n$ (the sample size) is large, the distribution of the $k \times 1$ vector of sample Sharpe ratios of each fund is well approximated by a multivariate normal distribution. In other words, if $n$ is large, we are justified in writing

$$
\widehat{\mathrm{SR}} \sim \mathrm{N}_{k}\left(\underline{\mathrm{SR}}, \frac{1}{n} \underline{\Omega}\right),
$$

where $\mathrm{N}_{k}$ refers to a multivariate normal distribution with $k$ variables and $\underline{\Omega}$ is equal to $D \Sigma D^{\mathrm{T}}$.

Journal of Risk 16(4) 
We wish to test the hypothesis that the Sharpe ratios of $k$ different funds are equal. Thus, our null hypothesis $H_{0}$ is the following:

$$
H_{0}: \mathrm{SR}_{1}=\mathrm{SR}_{2}=\cdots=\mathrm{SR}_{k}
$$

Let us define $(k-1) \times k$ contrast matrix $Q$ as

$$
Q=\left(\begin{array}{ccccc}
1 & -1 & 0 & \ldots & 0 \\
0 & 1 & -1 & \ldots & 0 \\
\vdots & & \ddots & \ddots & \vdots \\
0 & \ldots & \ldots & 1 & -1
\end{array}\right)
$$

and note that if the $k$ Sharpe ratios are equal, $Q \underline{S R}=\underline{0}$. Thus, a hypothesis test for equality of $k$ Sharpe ratios may be formulated as

$$
H_{0}: Q \underline{\mathrm{SR}}=\underline{0} \quad \text { versus } \quad H_{1}: Q \underline{\mathrm{SR}} \neq \underline{0} .
$$

According to Johnson and Wichern (2007) if $\underline{X}_{1}, \underline{X}_{2}, \ldots, \underline{X}_{n}$ is a sample from a multivariate normal distribution with $k$ variables, mean vector $\underline{\mu}$ and covariance matrix $\underline{\Sigma}$ we reject $H_{0}: \mu_{1}=\mu_{2}=\cdots=\mu_{k}$ if

$$
T^{2}=n\left(Q \bar{X}_{n}\right)^{\mathrm{T}}\left(Q \underline{\widehat{\Sigma}} Q^{\mathrm{T}}\right)^{-1}\left(Q \bar{X}_{n}\right)>\frac{(n-1)(k-1)}{(n-k+1)} F_{k-1, n-k+1}(\alpha),
$$

where $F_{k-1, n-k+1}(\alpha)$ is the upper $(100 \alpha)$ th percentile of an $F$-distribution with $k-1$ and $n-k+1$ degrees of freedom and $\underline{\widehat{\Sigma}}$ is the sample covariance matrix, the estimator for $\underline{\Sigma}$.

In our case we do not know for sure that the $\underline{X}_{i}$ follow a multivariate normal distribution. However, it is true that for large $n-k$,

$$
n\left(\bar{X}_{n}-\underline{\mu}\right)^{\mathrm{T}}(\underline{\widehat{\Sigma}})^{-1}\left(\bar{X}_{n}-\underline{\mu}\right) \text { is approximately } \chi_{k}^{2},
$$

which in turn implies that

$$
T^{2}=n\left(Q \bar{X}_{n}\right)^{\mathrm{T}}\left(Q \underline{\widehat{\Sigma}} Q^{\mathrm{T}}\right)^{-1}\left(Q \bar{X}_{n}\right) \text { is approximately } \chi_{k-1}^{2}
$$

and thus our $(100 \alpha) \%$-level hypothesis test for the equality of multiple Sharpe ratios under the assumption of iid returns is as follows:

$$
\text { Reject } H_{0}: \mathrm{SR}_{1}=\mathrm{SR}_{2}=\cdots=\mathrm{SR}_{k}
$$

if

$$
T^{2}=n(Q \underline{\widehat{\mathrm{SR}}})^{\mathrm{T}}\left(Q \underline{\hat{\Omega}} Q^{\mathrm{T}}\right)^{-1}(Q \underline{\widehat{\mathrm{SR}}})>\chi_{k-1}^{2}(\alpha),
$$


TABLE 1 Empirical rejection probabilities (\%) for Leung and Wong's (2008) test (LW) and the test described in this paper (WYY) when applied to iid returns generated from various multivariate distributions.

\begin{tabular}{|c|c|c|c|c|c|c|c|c|c|c|}
\hline \multirow[b]{3}{*}{ Distribution } & \multirow[b]{3}{*}{ Test } & \multicolumn{3}{|c|}{$k=5$} & \multicolumn{3}{|c|}{$k=10$} & \multicolumn{3}{|c|}{$k=20$} \\
\hline & & \multicolumn{3}{|c|}{$\alpha$} & \multicolumn{3}{|c|}{$\alpha$} & \multicolumn{3}{|c|}{$\alpha$} \\
\hline & & $1 \%$ & $5 \%$ & $10 \%$ & $1 \%$ & $5 \%$ & $10 \%$ & $1 \%$ & $5 \%$ & $10 \%$ \\
\hline \multirow[t]{2}{*}{ Normal } & LW & 0.7 & 4.5 & 9.5 & 1.1 & 5.2 & 10.1 & 1.1 & 5.0 & 10.1 \\
\hline & WYY & 0.7 & 4.7 & 9.7 & 1.2 & 5.6 & 10.9 & 1.4 & 6.2 & 11.9 \\
\hline \multirow{2}{*}{$t_{8}$} & LW & 2.8 & 10.2 & 17.2 & 3.8 & 12.7 & 21.3 & 5.0 & 16.1 & 25.9 \\
\hline & WYY & 1.1 & 5.4 & 10.8 & 1.5 & 6.2 & 12.0 & 1.6 & 6.8 & 12.7 \\
\hline \multirow[t]{2}{*}{$t_{6}$} & LW & 5.4 & 14.6 & 23.8 & 8.1 & 20.3 & 30.5 & 13.1 & 29.7 & 41.0 \\
\hline & WYY & 1.3 & 5.9 & 11.0 & 1.6 & 6.3 & 12.1 & 2.2 & 8.7 & 15.4 \\
\hline \multirow[t]{2}{*}{$t_{4}$} & LW & 24.1 & 39.4 & 49.2 & 39.4 & 56.4 & 65.9 & 58.9 & 74.7 & 82.3 \\
\hline & WYY & 2.1 & 8.0 & 13.9 & 3.5 & 10.6 & 18.1 & 6.8 & 17.3 & 26.9 \\
\hline
\end{tabular}

where $\chi_{k-1}^{2}$ is the upper $(100 \alpha)$ th percentile of a $\chi^{2}$-distribution with $k-1$ degrees of freedom and $\underline{\hat{\Omega}}$ is obtained from the sample covariance matrix and is the estimator for $\underline{\Omega}$.

As an illustration of the difference between the test advocated in this paper and that of Leung and Wong (2008), we conduct a simulation study similar to that in Ledoit and Wolf (2008), with the objective of computing empirical rejection probabilities under the null hypothesis. We simulate 2000 returns from $k$ assets from a multivariate distribution with mean vector $\mu$ and covariance matrix $V$. We take $\mu$ as a $k$-vector of ones and $V$ as a $k \times k$ matrix whose diagonal values are all one and off-diagonal values are all one half (these arbitrary values are extensions of the values used the simulation study of Ledoit and Wolf (2008)). We then apply Leung and Wong's (2008) test (LW) and our test (WYY) using the $T^{2}$ statistic given in (3.4) for the equality of the Sharpe ratios of the $k$ assets. The $p$-value of each test is recorded and we note whether the null hypothesis is rejected at the $1 \%, 5 \%$ and $10 \%$ levels.

The simulation and testing of returns is repeated 10000 times and the empirical rejection probabilities are recorded. The multivariate distributions we consider are the multivariate versions of the normal, $t_{4}, t_{6}$ and $t_{8}$ distributions. According to the theory detailed above, the WYY test should handle the nonnormal returns better than the LW test.

The results presented in Table 1 strongly suggest that this is the case. As a rule of thumb, the LW test is at least twice as likely to reject the null hypothesis at a given 
confidence level when the returns have heavy tails. The heavier the tails and the more assets under consideration, the greater the error of the LW test compared to the WYY test.

The test we derived in this section generalizes previous tests for the equality for multiple Sharpe ratios that were only suitable for multivariate normal iid returns. The WYY test holds for iid returns with finite fourth moments and although this extension is helpful, it falls short of being practically useful as in reality many returns exhibit time-series properties. We address this issue in the next section.

\section{A HYPOTHESIS TEST FOR THE EQUALITY OF MULTIPLE SHARPE RATIOS: NON-IID CASE}

As an empirical example of the applicability of their hypothesis test, Leung and Wong (2008) tested the equality of the Sharpe ratios of 17 iShares and Standard \& Poor's depository receipts which they treated as the 18th, American, iShare for comparison. They used the daily returns of all 18 securities from March 19, 1996 to December 31, 2003 (obtained from the Center for Research in Security Prices) and the three month T-bill (downloaded from Datastream) as the benchmark asset.

We have shown that their test requires returns to be iid with a multivariate normal distribution. Although the WYY test developed in Section 3 is an improvement, its requirement of iid returns may be overly restrictive. Autocorrelation, for example, is a noted feature of financial returns, but the WYY test, in its current version, cannot handle it.

Let us see if the iShares data used in Leung and Wong (2008) exhibits significant autocorrelation. In the univariate case, the Ljung-Box statistic (Ljung and Box 1978) tests the null-hypothesis of no autocorrelation in a time series. Its multivariate version, developed in Hosking (1980, 1981) and Li and McLoed (1981), tests the null hypothesis

$$
H_{0}: \underline{\rho}_{1}=\underline{\rho}_{2}=\cdots=\underline{\rho}_{m}=\underline{0}
$$

where $\underline{\rho}_{i}$ is the lag- $i$ cross-correlation matrix of the returns of $k$-assets, against the alternate hypothesis that $\underline{\rho}_{i} \neq \underline{0}$ for some $i \in\{1, \ldots, m\}$. The test statistic $Q(m)$ is calculated according to equation (6.1) of Hosking (1980). Applying this test to the iShares data set (choosing $m=8 \approx \ln (1961)$ ), we find that the multivariate series is strongly serially correlated. The results are shown in Table 2 on the next page.

Clearly any test assuming the iShares returns to be iid could produce misleading results. It behooves us to generalize the necessary conditions of our multiple Sharpe ratio test further, in order to at least provide a test that is safer for practitioners to use.

Two papers have already provided hypothesis tests for the Sharpe ratio under more general conditions. Lo (2002), in the section on non-iid returns, finds the asymptotic 
TABLE 2 Results of testing iShares returns for autocorrelation.

\begin{tabular}{lcccccccc}
\hline Lag $\boldsymbol{m}$ & $\mathbf{1}$ & $\mathbf{2}$ & $\mathbf{3}$ & $\mathbf{4}$ & $\mathbf{5}$ & $\mathbf{6}$ & $\mathbf{7}$ & $\mathbf{8}$ \\
\hline$Q(m)$ & 2115 & 2534 & 2969 & 3404 & 3841 & 4264 & 4696 & 5074 \\
$p$-value & 0 & 0 & 0 & 0 & 0 & 0 & 0 & 0 \\
\hline
\end{tabular}

distribution of a generalized method of moments (GMM) estimator of a single Sharpe ratio under the assumption that the distribution of the returns is stationary and ergodic (and also satisfies some regularity conditions). Section 3.1 of Ledoit and Wolf (2008) meanwhile, starts with the asymptotic distribution of the difference between two Sharpe ratios under similar general assumptions for the excess return and discusses various methods of estimating the limiting covariance matrix via heteroscedasticity and autocorrelation consistent (HAC) estimation. Both the GMM and HAC estimation methods can be readily extended to the case of multiple Sharpe ratios and thus we can weaken the assumptions required of the WYY test. We do so in this section.

The pattern for defining a test for the equality of multiple Sharpe ratios under more relaxed assumptions for the underlying excess return processes is the same as that in the previous section: find a multivariate asymptotic distribution for the estimators of interest; apply the multivariate delta method; use contrast matrixes to construct the hypothesis test. Lo (2002) begins by using the limiting distribution of the GMM estimator, as proven in Hansen (1982) and so shall we. Specifically, Theorem 3.1 of Hansen (1982) applies and we repeat it here.

TheOREM $4.1 \quad$ Stochastic process $\left\{\underline{Y}_{n}:-\infty<n<+\infty\right\}$ has $k$ components. $\&$ is a parameter space that is a subset of $\mathbb{R}^{q} . \underline{\beta}_{0}$ is the element of $\&$ we wish to estimate. We consider function $\underline{h}: \mathbb{R}^{p} \times \& \mapsto \mathbb{R}^{r}$, with $r \geqslant q$. Function $\underline{h}$ provides an expression for the $r$ orthogonality conditions that emerge from the parameters we wish to estimate. We make the following assumptions.

(1) $\left\{\underline{Y}_{n}:-\infty<n<+\infty\right\}$ is stationary and ergodic.

(2) $\&$ is an open subset of $\mathbb{R}^{q}$ that contains $\underline{\beta}_{0}$.

(3) $\underline{h}(\cdot, \beta)$ and $\partial \underline{h} / \partial \beta(\cdot, \beta)$ are Borel measurable for each $\beta \in \delta$ and $\partial \underline{h} / \partial \beta(\underline{Y}, \cdot)$ is continuous on \& for each $\underline{Y} \in \mathbb{R}^{p}$.

(4) $\partial \underline{h} / \partial \underline{\beta}\left(\underline{Y}_{1}, \cdot\right)$ is first moment continuous at $\underline{\beta}_{0}$, and $\mathbb{E}\left[\partial \underline{h} / \partial \underline{\beta}\left(\underline{Y}_{1}, \underline{\beta}_{0}\right)\right]$ exists, is finite, and has full rank. 
(5) Let $\left.\underline{w}_{n}=\underline{h}_{(} \underline{Y}_{n}, \underline{\beta}_{0}\right)$ for $-\infty<n<+\infty$ and

$$
\underline{v}_{j}=\mathbb{E}\left[\underline{w}_{0} \mid \underline{w}_{-j}, \underline{w}_{-j-1}, \ldots\right]-\mathbb{E}\left[\underline{w}_{0} \mid \underline{w}_{-j-1}, \underline{w}_{-j-2}, \ldots\right] \text { for } j \geqslant 0
$$

and assume

(a) $\mathbb{E}\left[\underline{w}_{0} \underline{w}_{0}^{\mathrm{T}}\right]$ exists and is finite;

(b) $\mathbb{E}\left[\underline{w} \mid \underline{w}_{-j}, \underline{w}_{-j-1}, \ldots\right]$ converges in mean square to zero;

(c) $\sum_{j=0}^{\infty} \mathbb{E}\left[\underline{v}_{j}^{\mathrm{T}} \underline{v}_{j}\right]^{1 / 2}$ is finite.

We also define the following terms.

- $\underline{g}_{N}(\underline{\beta}):=(1 / N) \sum_{n=1}^{N} \underline{h}\left(\underline{Y}_{n}, \underline{\beta}\right)$.

- Let $\left\{\underline{a}_{N}^{*}: N \geqslant 1\right\}$ be a sequence of $q$ by $r$ random matrixes that converges in probability to a constant matrix $\underline{a}_{0}^{*}$ which has full rank.

- Define the GMM estimator to be a sequence of random vectors $\left\{\underline{b}_{N}^{*}: N \geqslant 1\right\}$ that converges in probability to $\underline{\beta}_{0}$ for which the sequence $\left\{\sqrt{N} \underline{a}_{N}^{*} \underline{g}_{N}\left(\underline{b}_{N}^{*}\right)\right.$ : $N \geqslant 1$ \} converges in probability to zero.

- $\underline{d}_{0}:=\mathbb{E}\left[\partial \underline{h} / \partial \underline{\beta}\left(\underline{Y}_{1}, \underline{\beta}_{0}\right)\right]$.

- $\underline{R}_{w}(j):=\mathbb{E}\left[\underline{w}_{0} \underline{w}_{-j}^{\mathrm{T}}\right]$ and $\underline{S}_{w}:=\sum_{j=-\infty}^{+\infty} \underline{R}_{w}(j)$.

Supposing the assumptions above are satisfied, then $\left\{\sqrt{N}\left(\underline{b}_{N}^{*}-\underline{\beta}_{0}\right): N \geqslant 1\right\}$ converges in distribution to a normally distributed random vector with mean zero and covariance matrix $\left(\underline{a}_{0}^{*} \underline{d}_{0}\right)^{-1} \underline{a}_{0}^{*} \underline{S}_{w} \underline{a}_{0}^{* \mathrm{~T}}\left(\underline{a}_{0}^{*} \underline{d}_{0}\right)^{-1}$.

As Lo (2002) notes, Theorem 3.1 of Hansen (1982) allows us to consider the asymptotic distribution of estimators of parameters of excess return processes that exhibit non-iid characteristics such as "serial correlation, dependence on such factors as the market portfolio, time-varying conditional volatilities, jumps, and other empirically relevant phenomena".

Let us apply the above theorem for our purposes. As before, we define the $2 k \times 1$ vector $\underline{X}_{t}$ which has entries $X_{i t}$ for rows 1 to $k$ and $X_{i t}^{2}$ for rows $k+1$ to $2 k$. The parameters we wish to estimate are $m_{1}^{i}$ and $m_{2}^{i}$ for $i \in\{1, \ldots, k\}$, which are also the entries of the $2 k \times 1$ mean vector $\underline{\mu}$, the expected value of $\underline{X}_{t}$. Parameter space $\&$ is a subset of $\mathbb{R}^{2 k}$ that contains $\underline{\mu}$. Define vector function $\underline{h}$ as follows:

$$
\underline{h}: \mathbb{R}^{2 k} \times s \mapsto \mathbb{R}^{2 k}
$$

and for $\underline{x}=\left(x_{1}, \ldots, x_{2 k}\right)^{\mathrm{T}} \in \mathbb{R}^{2 k}$ and $\underline{\beta}=\left(\beta_{1}, \ldots, \beta_{2 k}\right)^{\mathrm{T}} \in \mathcal{s}$,

$$
h_{i}=x_{i}-\beta_{i} \quad \text { for } i=1, \ldots, 2 k .
$$


It is clear that $(\partial \underline{h} / \partial \beta)(\underline{Y}, \cdot)=-\underline{I}_{2 k}$ where $\underline{I}_{n}$ is the identity matrix of dimension $n$. Thus, assumptions (3) and (4) of the theorem above are trivially satisfied and in our case matrix $\underline{d}_{0}=-\underline{I}_{2 k}$. Using the notation of Section 2 , we easily see that $\underline{g}_{N}(\underline{\beta})=\bar{X}_{N}-\underline{\beta}$ and so by choosing $\underline{a}_{N}^{*}=\underline{I}_{2 k}$ and $\underline{b}_{N}^{*}=\bar{X}_{N}$ for all $N$, we satisfy the conditions required of random matrix sequence $\left(\underline{a}_{N}^{*}\right)$ and GMM estimator $\left(\underline{b}_{N}^{*}\right)$. Thus, assuming that our excess returns satisfy assumptions (1) and (5) we have that

$$
\sqrt{n}\left(\bar{X}_{n}-\underline{\mu}\right) \stackrel{\mathrm{D}}{\rightarrow} \mathrm{N}\left(\underline{0}, \underline{S}_{w}\right) .
$$

Recall vector function $f$ defined in (2.4). As in Section 2, we may apply the multivariate delta method with $\underline{f}$ to find the asymptotic distribution of $\underline{\widehat{S R}}$, the vector of estimates of Sharpe ratios for the $k$ funds:

$$
\sqrt{n}(\underline{\widehat{\mathrm{SR}}}-\underline{\mathrm{SR}}) \stackrel{\mathrm{D}}{\rightarrow} \mathrm{N}\left(\underline{0}, D \underline{S}_{w} D^{\mathrm{T}}\right),
$$

where $D$ is the $k \times 2 k$ matrix defined in (2.10). From this, we formulate a hypothesis test for the equality of the Sharpe Ratios of $k$ funds in the same fashion as Section 3:

$$
\text { Reject } H_{0}: \mathrm{SR}_{1}=\mathrm{SR}_{2}=\cdots=\mathrm{SR}_{k}
$$

if

$$
T^{2}=n(Q \underline{\widehat{\mathrm{SR}}})^{\mathrm{T}}\left(Q \underline{\hat{\Omega}} Q^{\mathrm{T}}\right)^{-1}(Q \underline{\widehat{\mathrm{SR}}})>\chi_{k-1}^{2}(\alpha),
$$

where $\chi_{k-1}^{2}$ is the upper $(100 \alpha)$ th percentile of an $\chi^{2}$-distribution with $k-1$ degrees of freedom, $Q$ is the contrast matrix defined in 3.2 and $\underline{\hat{\Omega}}$ is an estimator for $D \underline{S}_{w} D^{\mathrm{T}}$.

Application of the test thus requires estimation of matrixes $D$ and $\underline{S}_{w}$. Matrix $D$ can be estimated by replacing the moments of the excess return that appear in its definition by the sample means of the excess returns and their squares. Finding a consistent estimator for covariance matrix $\underline{S}_{w}$ however requires a more sophisticated technique. Lo (2002) uses Newey and West's (1987) procedure. Section 3.1 of Ledoit and Wolf (2008) provides a good summary of HAC estimators of covariance matrixes, of which there are many. We shall follow Lo (2002) and use the Newey-West estimator for $\underline{S}_{w}$, our estimator for $\underline{S}_{w}$. Thus for our purposes the Newey-West estimator is given by

$$
\hat{\underline{S}_{w}}=\underline{\hat{V}}_{0}+\sum_{j=1}^{m}\left(1-\frac{j}{m+1}\right)\left[\hat{\hat{V}}_{j}+\underline{\hat{V}}_{j}^{\mathrm{T}}\right] \text {, }
$$

where

$$
\left.\left.\underline{\hat{V}}_{j}=\frac{1}{N} \sum_{t=j+1}^{N} \underline{h}_{t}, \underline{X}_{N}\right) \underline{h}_{\left(\underline{X}_{t}\right.}, \bar{X}_{N}\right)^{\mathrm{T}} .
$$

We shall choose $m=\left\lfloor N^{1 / 3}\right\rfloor$, as suggested in Newey and West (1994). 
TABLE 3 Results of applying the three tests to the iShares data.

\begin{tabular}{cccc}
\hline Year & $\begin{array}{c}\text { LW } \\
\boldsymbol{p} \text {-value }\end{array}$ & $\begin{array}{c}\text { WYY iid } \\
\boldsymbol{p} \text {-value }\end{array}$ & $\begin{array}{c}\text { WYY non-iid } \\
\boldsymbol{p} \text {-value }\end{array}$ \\
\hline 1996 & 0.880 & 0.836 & 0.416 \\
1997 & 0.421 & 0.460 & 0.253 \\
1998 & 0.919 & 0.906 & 0.464 \\
1999 & 0.546 & 0.490 & 0.049 \\
2000 & 0.992 & 0.991 & 0.802 \\
2001 & 0.992 & 0.991 & 0.842 \\
2002 & 1 & 1 & 0.994 \\
2003 & 0.987 & 0.983 & 0.867 \\
All & 0.998 & 0.998 & 0.974 \\
years & & & \\
\hline
\end{tabular}

We are now in a position to apply the test derived in this section (WYY-non-iid) to the iShares data set. For comparison we, also apply Leung and Wong's (2008) test and the test derived in Section 3. Thus, we shall use exactly the same set of data as Leung and Wong (2008): 1961 daily (and therefore excess) returns for each of the 18 securities. The excess return of security $i$ on day $t, d_{i t}$, was calculated by subtracting that day's daily return on the three month T-bill, $r_{t}$, from the daily return without dividends of security $i, y_{i t}$. Thus each iShare has 1961 excess returns, from which their estimated Sharpe ratio is calculated as follows: ${ }^{1}$

$$
\widehat{\mathrm{SR}}_{i}=\frac{[1 / 1961] \sum_{t=1}^{1961} d_{i t}}{\sqrt{[1 / 1961] \sum_{t=1}^{1961} d_{i t}^{2}-\left([1 / 1961] \sum_{i=1}^{1961} d_{i t}\right)^{2}}} .
$$

For further comparison with Leung and Wong (2008), we calculate a test for equality of the Sharpe ratios for each year in the sample, making the corresponding changes to our estimators, as well as for the whole sample. The results are summarized in Table 3.

${ }^{1}$ Leung and Wong (2008) use

$$
\widehat{\mathrm{SR}}_{i}=\frac{[1 / 1961] \sum_{t=1}^{1961} y_{i t}-r_{f}}{\sqrt{[1 / 1961] \sum_{t=1}^{1961} y_{i t}^{2}-\left([1 / 1961] \sum_{t=1}^{1961} y_{i t}\right)^{2}}}
$$

for each iShare $i$, where $r_{f}$ is "the three-month T-bill rate". However, this benchmark return varies over time so it is unsure as to what value $r_{f}$ takes. Our estimator for $\mathrm{SR}_{i}$ is the modified estimator recommended in Sharpe (1994) as it takes into account the possibility that the benchmark rate of return may change and emphasizes the importance of the differential (or "excess") return, $d$. 
Firstly, we note the contradiction between the results of Leung and Wong (2008) which strongly rejected the null hypothesis of equality and the results of our application of their test (LW) in Table 3 on the preceding page. With high $p$-values for each year and for the entire sample, we are unable to reject the null hypothesis that the Sharpe ratios of the iShares are equal. We used a slightly different definition of the Sharpe ratio to Leung and Wong (2008) (see footnote 1), but it is unlikely that this explains the discrepancy. Our findings agree with the finding of Gasbarro et al (2007), who tested the equality of each possible pair of Sharpe ratios individually using the methods derived in Jobson and Korkie (1981) and Memmel (2003) and found that the null hypothesis of equality could not be rejected for any of them.

Secondly, it is interesting to note the similarity of the $p$-values given by the LW and WYY-iid tests. Finally, application of the test derived in this section would reject the null hypothesis at the 5\% level for one year only. Although the $p$-values given by the WYY-non-iid test are noticeably different to those of the other two tests, they are not small enough for us to infer that the Sharpe ratios of the iShares are not the same.

\section{CONCLUSIONS}

The Sharpe ratio is an often used indicator of an asset's worth as an investment. Statistical procedures designed for the purpose of testing whether Sharpe ratios of various funds or securities are equal are therefore of practical interest. Research has been conducted in this area, but much of it has focussed on pairwise tests of equality or has assumed that returns to be normal in nature (Jobson and Korkie 1981 and Memmel 2003). Leung and Wong (2008) sought to apply the repeated measures design (see Johnson and Wichern 2007, Chapter 6) and derive a hypothesis test for the equality of multiple Sharpe ratios under the more general assumption that returns are independently and identically distributed. However, their asymptotic distribution of the vector of Sharpe ratio estimates only holds true when returns are normally distributed and their application of their test to 17 iShares and Deposit Receipts uses an old estimator for the Sharpe ratio.

This paper extends the work of Leung and Wong (2008) by finding the asymptotic distribution of multiple Sharpe ratios firstly under the assumption of independently and identically distributed returns and secondly under the more general assumption that the returns are stationary and ergodic. It is shown that when returns are assumed to be normal, the distribution boils down to that in Leung and Wong (2008). Using these new hypothesis tests, we repeated the empirical example of Leung and Wong (2008), testing for the equality of the Sharpe ratios of 18 securities. The results suggest that the hypothesis that the Sharpe ratios are all the same cannot be rejected at the $1 \%$ level, in contrast to the main finding of Leung and Wong (2008). 
It is hoped that our test may be of practical value to practitioners. Future versions could include different HAC estimators for the limiting covariance matrix. Alternatively, an alternate test under general assumptions could be derived via the time series bootstrap approach developed in Ledoit and Wolf (2008).

\section{APPENDIX A. CALCULATING THE ENTRIES OF COVARIANCE MATRIX $D \underline{\Sigma} D^{\mathrm{T}}$}

Firstly we show that our results under the iid assumption replicate the known asymptotic distribution when $k=1$. The asymptotic variance in this case has been proven by Bentkus et al (2007). In that paper, the authors derived the limiting distribution of the noncentral $t$ statistic $\mathbb{T}_{n}$ defined by

$$
\mathbb{T}_{n}=\frac{\sqrt{n} \bar{X}}{S_{n}},
$$

where $\bar{X}$ and $S_{n}$ are the sample mean and sample standard deviation respectively of a sequence $X_{1}, X_{2}, \ldots, X_{n}$ of independent and identically distributed random variables. We rewrite their Theorem 2.1 below.

Theorem A 1 Let $X_{1}, \ldots, X_{n}, \bar{X}, S_{n}, \mathbb{T}_{n}$ be as described above. Let $X$ be an independent copy of $X_{1}$. Assume $\mathbb{E}\left[X^{4}\right]<\infty$. Then, unless $X$ is a specific linear function of a standardized Bernoulli random variable, we have

$$
\sigma_{0}^{-1}\left(\mathbb{T}_{n}-\sqrt{n} \frac{\mu}{\sigma}\right) \stackrel{\mathrm{D}}{\rightarrow} N(0,1)
$$

where $\stackrel{\mathrm{D}}{\rightarrow}$ denotes convergence in distribution, $\mu=\mathbb{E}[X], \sigma=\operatorname{Var}[X], \gamma_{3}$ is the skewness of $X, \gamma_{4}$ is the (excess) kurtosis of $X$ and finally

$$
\sigma_{0}^{2}=1-\frac{\mu \gamma_{3}}{\sigma}+\frac{\mu^{2} \gamma_{4}}{4 \sigma^{2}}+\frac{\mu^{2}}{2 \sigma^{2}}
$$

Referring to our paper, if $k=1$, then matrix $D$ is a $1 \times 2$ matrix:

$$
D=\left(\frac{m_{2}}{\left(m_{2}-\left(m_{1}\right)^{2}\right)^{3 / 2}} \frac{-m_{1}}{2\left(m_{2}-\left(m_{1}\right)^{2}\right)^{3 / 2}}\right),
$$

where $m_{j}=\mathbb{E}\left[X^{j}\right]$, the $j$ th raw moment of the return of the single asset under construction. Matrix $\underline{\Sigma}$, the asymptotic covariance for the mean vector $\left(\hat{m}_{1} \hat{m}_{2}\right)^{\mathrm{T}}$, is given by

$$
\underline{\Sigma}=\left(\begin{array}{cc}
m_{2}-m_{1}^{2} & m_{3}-m_{1} m_{2} \\
m_{3}-m_{1} m_{2} & m_{4}-m_{2}^{2}
\end{array}\right) \text {. }
$$


Calculating $D \underline{\Sigma} D^{\mathrm{T}}$ we find the asymptotic variance of the Sharpe ratio estimator $\widehat{\mathrm{SR}}$ to be

$$
\sigma_{1}^{2}:=\left(4 m_{2}^{3}-m_{1}^{2} m_{2}^{2}-4 m_{1} m_{2} m_{3}+m_{1}^{2} m_{4}\right) / 4 \sigma^{3},
$$

where $\sigma$ is the standard deviation of return $X$. As stated previously, Bentkus et al (2007) prove the asymptotic variance of the estimator to be

$$
\sigma_{0}^{2}:=1-m_{1} \gamma_{3} / \sigma+m_{1}^{2} \gamma_{4} / \sigma^{2}-m_{1}^{2} /\left(4 \sigma^{2}\right)
$$

where $\gamma_{3}$ and $\gamma_{4}$ are the skewness and kurtosis of return $X$. By expressing $\gamma_{3}$ and $\gamma_{4}$ in terms of raw moments $m_{1}, m_{2}, m_{3}$ and $m_{4}$ as

$$
\begin{aligned}
& \gamma_{3}=\left(2 m_{1}^{3}-3 m_{1} m_{2}+m_{3}\right) / \sigma^{3}, \\
& \gamma_{4}=\left(-3 m_{1}^{4}+6 m_{1}^{2} m_{2}-4 m_{1} m_{3}+m_{4}\right) / \sigma^{4}
\end{aligned}
$$

and plugging these into Bentkus et al's variance, we recover $\sigma_{1}^{2}$. Thus, our asymptotic variance $D \underline{\Sigma} D^{\mathrm{T}}$ recovers existing results in the case when $k=1$.

We shall now calculate the entries of our covariance matrix $D \underline{\Sigma} D^{\mathrm{T}}$ explicitly and see how they compare to the entries of the covariance matrix $\Omega$ found in Leung and Wong (2008). If $D=\left[D_{1}: D_{2}\right]$ then it follows that

$$
\begin{aligned}
D \underline{\Sigma} D^{\mathrm{T}} & =\left(\begin{array}{ll}
D_{1} & D_{2}
\end{array}\right)\left(\begin{array}{cc}
A & C \\
C^{\mathrm{T}} & B
\end{array}\right)\left(\begin{array}{l}
D_{1} \\
D_{2}
\end{array}\right) \\
& =D_{1} A D_{1}+D_{2} C^{\mathrm{T}} D_{1}+D_{1} C D_{2}+D_{2} B D_{2} .
\end{aligned}
$$

Matrix $A$ is the covariance matrix of the excess returns of each fund. Thus the $(i, j)$ th entry of $A, a_{i j}$ is $\operatorname{Cov}\left(X_{i t}, X_{j t}\right):=\sigma_{i j}$. Since the $i$ th diagonal entry of $D_{1}$ is equal to $\left(\sigma_{i}^{2}+\mu_{i}^{2}\right) / \sigma_{i}^{3}$ where $\sigma_{i}$ is the standard deviation of the excess return of fund $i$ and $\mu_{i}$ is its mean, simple calculations show that the diagonal terms of the matrix $D_{1} A D_{1}$ are given by

$$
\left(D_{1} A D_{1}\right)_{i i}=\frac{\sigma_{i}^{2}+\mu_{i}^{2}}{\sigma_{i}^{3}} \sigma_{i}^{2} \frac{\sigma_{i}^{2}+\mu_{i}^{2}}{\sigma_{i}^{3}}=\frac{\left(\sigma_{i}^{2}+\mu_{i}^{2}\right)^{2}}{\sigma_{i}^{4}}
$$

and the off-diagonal terms are given by

$$
\begin{aligned}
\left(D_{1} A D_{1}\right)_{i j} & =\frac{\sigma_{i}^{2}+\mu_{i}^{2}}{\sigma_{i}^{3}} \sigma_{i j} \frac{\sigma_{j}^{2}+\mu_{j}^{2}}{\sigma_{j}^{3}} \\
& =\frac{\left(\sigma_{i}^{2}+\mu_{i}^{2}\right)\left(\sigma_{j}^{2}+\mu_{j}^{2}\right) \sigma_{i j}}{\sigma_{i}^{3} \sigma_{j}^{3}}=\left(D_{1} A D_{1}\right)_{j i} .
\end{aligned}
$$

Matrix $B$ is the covariance matrix for the squared excess returns of each fund. We shall denote the $(i, j)$ th entry of $B, \operatorname{Cov}\left(X_{i t}^{2}, X_{j t}^{2}\right)$, by $s_{i j}$ and the standard deviation 
of $X_{i t}^{2}$ by $s_{i}$. The $i$ th diagonal entry of $D_{2}$ is equal to $-\mu_{i} / 2 \sigma_{i}^{3}$, simple calculation reveal that the diagonal entries of the matrix $D_{2} B D_{2}$ are given by

$$
\left(D_{2} B D_{2}\right)_{i i}=\frac{-\mu_{i}}{2 \sigma_{i}^{3}} s_{i}^{2} \frac{\mu_{i}}{2 \sigma_{i}^{3}}=\frac{\mu_{i}^{2} s_{i}^{2}}{4 \sigma_{i}^{6}}
$$

and the off-diagonal terms are given by

$$
\left(D_{2} B D_{2}\right)_{i j}=\frac{-\mu_{i}}{2 \sigma_{i}^{3}} s_{i j} \frac{\mu_{i}}{2 \sigma_{i}^{3}}=\frac{\mu_{i} \mu_{j} s_{i j}}{4 \sigma_{i}^{3} \sigma_{j}^{3}}=\left(D_{2} B D_{2}\right)_{j i} .
$$

Matrix $C$ is the covariance matrix of the $k$ excess returns against the $k$ squared excess returns. We shall denote the $(i, j)$ th entry of $C, \operatorname{Cov}\left(X_{i t}, X_{j t}^{2}\right)$, by $q_{i j}$ and $\operatorname{Cov}\left(X_{i t}, X_{i t}^{2}\right)$ by $q_{i}^{2}$. Again, tedious calculations show that the diagonal entries of $D_{1} C D_{2}$ are given by

$$
\left(D_{1} C D_{2}\right)_{i i}=\frac{\sigma_{i}^{2}+\mu_{i}^{2}}{\sigma_{i}^{3}} q_{i}^{2} \frac{-\mu_{i}}{2 \sigma_{i}^{3}}=\frac{-\mu_{i}\left(\sigma_{i}^{2}+\mu_{i}^{2}\right) q_{i}^{2}}{2 \sigma_{i}^{6}}
$$

and the off-diagonal terms are

$$
\left(D_{1} C D_{2}\right)_{i j}=\frac{\sigma_{i}^{2}+\mu_{i}^{2}}{\sigma_{i}^{3}} q_{i j} \frac{-\mu_{j}}{2 \sigma_{j}^{3}}=\frac{-\mu_{j}\left(\sigma_{i}^{2}+\mu_{i}^{2}\right) q_{i j}}{2 \sigma_{i}^{3} \sigma_{i}^{3}} .
$$

Since $D_{2} C^{\mathrm{T}} D_{1}$ is the transpose of $D_{1} C D_{2}$, its entries are easily found and we find the entries of the symmetric matrix $D_{2} C^{\mathrm{T}} D_{1}+D_{1} C D_{2}$ as follows: on the diagonal we have

$$
\left(D_{2} C^{\mathrm{T}} D_{1}+D_{1} C D_{2}\right)_{i i}=\frac{-\mu_{i}\left(\sigma_{i}^{2}+\mu_{i}^{2}\right) q_{i}^{2}}{\sigma_{i}^{6}}
$$

and off the diagonal we have

$$
\left(D_{2} C^{\mathrm{T}} D_{1}+D_{1} C D_{2}\right)_{i j}=\frac{-\mu_{j}\left(\sigma_{i}^{2}+\mu_{i}^{2}\right) q_{i j}-\mu_{i}\left(\sigma_{j}^{2}+\mu_{j}^{2}\right) q_{j i}}{2 \sigma_{i}^{3} \sigma_{j}^{3}} .
$$

Summing the matrixes together, we find that the diagonal entries of our asymptotic covariance matrixes are

$$
\left(D \underline{\Sigma} D^{\mathrm{T}}\right)_{i i}=\frac{4 \sigma_{i}^{2}\left(\sigma_{i}^{2}+\mu_{i}^{2}\right)^{2}+\mu_{i}^{2} s_{i}^{2}-4 \mu_{i}\left(\sigma_{i}^{2}+\mu_{i}^{2}\right) q_{i}^{2}}{4 \sigma_{i}^{6}}
$$

and the nondiagonal entries are

$$
\begin{aligned}
\left(D \underline{\Sigma} D^{\mathrm{T}}\right)_{i j}=\frac{1}{4 \sigma_{i}^{3} \sigma_{j}^{3}}\left(4\left(\sigma_{i}^{2}+\mu_{i}^{2}\right)\left(\sigma_{j}^{2}+\mu_{j}^{2}\right) \sigma_{i j}+\mu_{i} \mu_{j} s_{i j}\right. & \\
& \left.-2 \mu_{j}\left(\sigma_{i}^{2}+\mu_{i}^{2}\right) q_{i j}-2 \mu_{i}\left(\sigma_{j}^{2}+\mu_{j}^{2}\right) q_{j i}\right) .
\end{aligned}
$$


Let us calculate $D \underline{\Sigma} D^{\mathrm{T}}$ under the assumption that the excess daily returns for the $k$ funds follow a multivariate normal distribution, so that the daily excess return for fund $i$ has mean $\mu_{i}$, standard deviation $\sigma_{i}$ and covariance $\sigma_{i j}$ with the daily excess return of fund $j$. By the formula for the raw moments of the normal distribution, we know that

$$
s_{i}^{2}=2 \sigma_{i}^{4}+4 \mu_{i}^{2} \sigma_{i}^{2} \quad \text { and } \quad q_{i}^{2}=2 \mu_{i} \sigma_{i}^{2} .
$$

After plugging these values into $\left(D \underline{\Sigma} D^{\mathrm{T}}\right)_{i i}$, we see that the diagonal entries of the asymptotic covariance matrix when the excess returns are multivariate normal

$$
\left(D \underline{\Sigma} D^{\mathrm{T}}\right)_{i i}=1+\frac{\mu_{i}^{2}}{2 \sigma_{i}^{2}}=1+\frac{1}{2} \mathrm{SR}_{i}^{2} .
$$

To find the off-diagonal terms in the normal case, we need to express $s_{i j}$ and $q_{i j}$ in terms of the parameters of the multivariate normal distribution, $\mu_{i}, \sigma_{i}$ and covariance $\sigma_{i j}$. To that end, we use Isserlis's theorem (Isserlis 1918), from which it follows that

$\mathbb{E}\left[\left(X_{i t}-\mu_{i}\right)\left(X_{j t}-\mu_{j}\right)^{2}\right]=0 \quad$ and $\quad \mathbb{E}\left[\left(X_{i t}-\mu_{i}\right)^{2}\left(X_{j t}-\mu_{j}\right)^{2}\right]=\sigma_{i}^{2} \sigma_{j}^{2}+2\left(\sigma_{i j}\right)^{2}$.

Expanding and rearranging the above we find two relations:

$$
\begin{aligned}
\mathbb{E}\left[X_{i t} X_{j t}^{2}\right]= & 2 \mu_{j} \sigma_{i j}+\mu_{i} \sigma_{j}^{2}+\mu_{i} \mu_{j}^{2}, \\
\mathbb{E}\left[X_{i t}^{2} X_{j t}^{2}\right]= & 2 \mu_{i} \mathbb{E}\left[X_{i t} X_{j t}^{2}\right]+2 \mu_{j} \mathbb{E}\left[X_{j t} X_{i t}^{2}\right] \\
& -4 \mu_{i} \mu_{j} \sigma_{i j}-\sigma_{i}^{2} \mu_{j}^{2}-\mu_{i}^{2} \sigma_{j}^{2}-3 \mu_{i}^{2} \mu_{j}^{2}+\sigma_{i}^{2} \sigma_{j}^{2}+2\left(\sigma_{i j}\right)^{2},
\end{aligned}
$$

and after yet more calculations we find

$$
s_{i j}=2\left(\sigma_{i j}\right)^{2}+4 \mu_{i} \mu_{j} \sigma_{i j} \text { and } q_{i j}=2 \mu_{j} \sigma_{i j} .
$$

Inserting these into the expression for the nondiagonal entries reveals

$$
(D \underline{\Sigma} D)_{i j}=\rho_{i j}+\frac{1}{2} \mathrm{SR}_{i} \mathrm{SR}_{j} \rho_{i j}^{2},
$$

where $\rho_{i j}$ is the correlation between the excess returns of funds $i$ and $j$. Thus $(D \underline{\Sigma} D)_{i j}$, when excess returns are assumed to be normally distributed, is equal to the asymptotic covariance matrix found in Leung and Wong (2008).

\section{REFERENCES}

Bentkus, V., Jing, B.-Y., Shao, Q.-M., and Zhou, W. (2007). Limiting distributions of the noncentral $t$-statistic and their applications to the power of $t$-tests under nonnormality. Bernoulli 13(2), 346-364.

Gasbarro, D., Wong, W.-K., and Kenton Zumwalt, J. (2007). Stochastic dominance analysis of iShares. European Journal of Finance 13(1), 89-101. 
Hansen, P. L. (1982). Large sample properties of generalized method of moments estimators. Econometrica 50(4), 1029-1054.

Hosking, J. R. M. (1980). The multivariate portmanteau statistic. Journal of the American Statistical Association 75(371), 602-608.

Hosking, J. R. M. (1981). Lagrange-multiplier tests of multivariate time-series models. Journal of the Royal Statistical Society B 43(2), 219-230.

Isserlis, L. (1918). On a formula for the product-moment coefficient of any order of a normal frequency distribution in any number of variables. Biometrika 12(1-2), 134-139.

Jobson, J. D., and Korkie, B. M. (1981). Performance hypothesis testing with the Sharpe and Treynor measures. Journal of Finance 36(4), 889-908.

Johnson, R. A., and Wichern, D. W. (2007). Applied Multivariate Statistical Analysis. Pearson Prentice Hall.

Ledoit, O., and Wolf, M. (2008). Robust performance hypothesis testing with the sharpe ratio. Journal of Empirical Finance 15(5), 850-859.

Leung, P.-L., and Wong, W.-K. (2008). On testing the equality of multiple Sharpe ratios, with application on the evaluation of iShares. The Journal of Risk 10(3), 15-30.

Li, W. K., and McLeod, A. I. (1981). Distribution of the residual autocorrelations in multivariate arma time series models. Journal of the Royal Statistical Society B 43(2), 231239.

Ljung, G. M., and Box, G. E. P. (1978). On a measure of lack of fit in time series models. Biometrika 65(2), 297-303.

Lo, A. W. (2002). The statistics of Sharpe ratios. Financial Analysts Journal 58(4), 36-52.

Memmel, C. (2003). Performance hypothesis testing with the Sharpe ratio. Finance Letters 1, 21-23.

Newey, W. K., and West, K. D. (1987). A simple positive semi-definite heteroscedasticity and autocorrelation consistent covariance matrix. Econometrica 55(3), 703-708.

Newey, W. K., and West, K. D. (1994). Automatic lag selection in covariance matrix estimation. Review of Economic Studies 61(4), 631-653.

Sharpe, W. F. (1994). The Sharpe ratio. Journal of Portfolio Management 21(1), 49-58.

Sharpe, W. F. (1966). Mutual fund performance. Journal of Business 39(1), 119-138. 
